\title{
Slow-theta power decreases during item-place encoding predict spatial accuracy of subsequent context recall
}

\author{
Maité Crespo-García ${ }^{\mathrm{a}, \mathrm{b}, *}$, Monika Zeiller ${ }^{\mathrm{a}}$, Claudia Leupold ${ }^{\mathrm{d}}$, Gernot Kreiselmeyer ${ }^{\mathrm{c}}$, Stefan Rampp ${ }^{\mathrm{d}}$, \\ Hajo M. Hamer ${ }^{c}$, Sarang S. Dalal ${ }^{\text {a,b }}$ \\ a Department of Psychology, University of Konstanz, 78464 Konstanz, Germany \\ b Zukunftskolleg, University of Konstanz, 78464 Konstanz, Germany \\ c Department of Neurology, Epilepsy Center, University Hospital Erlangen, 91054 Erlangen, Germany \\ d Department of Neurosurgery, Epilepsy Center, University Hospital Erlangen, 91054 Erlangen, Germany
}

\begin{abstract}
A B S T R A C T
Human hippocampal theta oscillations play a key role in accurate spatial coding. Associative encoding involves similar hippocampal networks but, paradoxically, is also characterized by theta power decreases. Here, we inves tigated how theta activity relates to associative encoding of place contexts resulting in accurate navigation. Using MEG, we found that slow theta $(25 \mathrm{~Hz})$ power negatively correlated with subsequent spatial accuracy for vir tual contextual locations in posterior hippocampus and other cortical structures involved in spatial cognition. A rare opportunity to simultaneously record MEG and intracranial EEG in an epilepsy patient provided crucial in sights: during power decreases, slow theta in right anterior hippocampus and left inferior frontal gyrus phase led the left temporal cortex and predicted spatial accuracy. Our findings indicate that decreased slow theta activ ity reflects local and long range neural mechanisms that encode accurate spatial contexts, and strengthens the view that local suppression of low frequency activity is essential for more efficient processing of detailed information.
\end{abstract}

Keywords:

Hippocampus

Spatial navigation

MEG

Intracranial EEG

Source localization

\section{Introduction}

A major goal of human memory research is to elucidate the neural mechanisms underlying memory formation. Explorations of the subse quent memory effect (SME), initially examined by contrasting hemody namic responses during the encoding of later remembered events with those of later forgotten events, have identified many of the neural corre lates of successful encoding (Schacter and Wagner, 1999). Applying this contrast to electrophysiological activity has additionally revealed that SMEs are associated with specific frequency bands, helping to delineate how encoding processes are regulated by oscillatory activity. Among these frequencies, the $48 \mathrm{~Hz}$ band received special attention in humans because it resembles the theta rhythm of small mammals, which has ex tensively shown memory related properties (Buzsáki and Moser, 2013; Hasselmo, 2005). For example, in rabbits, hippocampal theta activity pre dicts the learning rate of classical conditioning, whereas its loss impairs spatial memory in rats (Winson, 1978). In addition to these observations, it was found that theta bursts can induce long term potentiation (LTP) of synapses in hippocampus (Larson et al., 1986; Larson and Munkácsy,

\footnotetext{
* Corresponding author at: Department of Psychology, University of Konstanz, Germany.

E-mail address: maite.crespo-garcia@uni-konstanz.de (M. Crespo-García).
}

2015), suggesting a physiological mechanism by which theta oscillations could mediate memory storage processes.

In agreement with animal literature, human EEG/MEG studies have often found that theta power during encoding is positively correlated with subsequent memory (Atienza et al., 2011; Crespo Garcia et al., 2012; Friese et al., 2013; Hanslmayr et al., 2009; Klimesch et al., 1996; Osipova et al., 2006; Summerfield and Mangels, 2005). These positive SMEs were usually seen over frontal and temporal regions and corroborated by intracranial EEG (iEEG) recordings (Lega et al., 2012; Sederberg et al., 2003). It has been proposed that theta power increases during successful encoding might reflect the recruitment of cortical regions (Sederberg et al., 2003), where theta oscillations could be induced via hippocampo cortical feedback loops (Klimesch et al., 1996). In this way, human theta oscillations could modulate synaptic plasticity across hippocampus and cortex via a phase dependent mechanism (Crespo Garcia et al., 2010; Osipova et al., 2006; Sederberg et al., 2003) similar to the one demonstrated in the rat hippocampus (Hölscher et al., 1997; Pavlides et al., 1988).

However, other studies have reported negative SMEs in the broad theta band (Burke et al., 2013; Guderian et al., 2009; Long et al., 2014; Sederberg et al., 2007). These theta power decreases are seen during the successful encoding of single items and inter item associations 
(Greenberg et al., 2015) and are widely detected with surface sensors or iEEG contacts targeting medial temporal lobe (MTL) and fronto temporal structures (Greenberg et al., 2015; Long et al., 2014). Since negative SMEs manifest in key regions for successful encoding (e.g., hippocampus and left prefrontal cortex), the traditional vision that increased theta activity is necessary for memory formation faces a challenge. To explain these findings, Greenberg and collaborators (Greenberg et al., 2015) proposed that theta power decreases may reflect reduced neuronal correlations across the cortex and MTL. Such a mechanism would be beneficial for episodic memory encoding because it means that the network is able to process and store more information.

Although these contrary theta patterns are not fully understood, some experiments have shown that SMEs can be dissociated by specific task manipulations or other oscillatory properties. Positive or negative SMEs manifest when study and test contexts match or mismatch, re spectively, which constitutes evidence that theta power plays a crucial role in context item binding (Staudigl and Hanslmayr, 2013). SMEs also vary across brain regions, time windows and frequency ranges (e.g., early narrow band frontal power increases vs. late broad band hippocampal power decreases; Long et al., 2014) and seem to reflect different phase synchronization patterns (Burke et al., 2013). Besides these parameters, opposite SMEs can coexist in hippocampus at distinct frequency components (positive SMEs at $\sim 3 \mathrm{~Hz}$ vs. negative SMEs at $\sim 8 \mathrm{~Hz}$; Lega et al., 2012), suggesting the existence of functionally disso ciable theta bands (for more details, see discussion in Lega et al., 2012). Indeed, growing evidence indicates that slow theta activity (peaking at $\sim 3 \mathrm{~Hz}$ ) in the human hippocampus may be the functional equivalent to the hippocampal theta rhythm of rodents (Jacobs, 2014; Watrous et al., 2013). For example, when previous findings in rats (Vanderwolf, 1969) were replicated in neurosurgical patients with depth recordings in hip pocampus, it was observed that activity in slower frequencies $(14 \mathrm{~Hz})$ increased with movement in virtual navigation (Clemens et al., 2013; Ekstrom et al., 2005; Watrous et al., 2011). In addition, MEG studies have shown that better navigation performance is related to increased hippocampal activity at slow theta frequencies (Cornwell et al., 2008; Kaplan et al., 2012).

Together, these findings suggest that human hippocampal slow theta activity may be involved in the formation of accurate contextual spatial locations supporting spatial navigation. To investigate this hypothesis and the sign of the relationship, we directly focused on theta activity time locked to the encoding of item place associations, and correlated it with spatial accuracy for contextual locations in a subsequent memory test. We designed a spatial navigation video game to implement a SME experiment. The game was set in a computer simulated grass arena, with distal cues comprising a mountain landscape and clouds. The arena was populated with dif ferent pictures of buildings at given locations that participants were instructed to remember. In a later session, participants were prompted with specific building pictures, and asked to either orient themselves in the direction of the building or navigate to the remem bered location. The successful performance of the task partly relied on the formation of associations between the pictures and distal visual cues, as well as accurate spatial information derived from path integration mechanisms, two processes in which hippocampus is known to be involved (O'Keefe and Nadel, 1978). Theta SMEs were evaluated by performing correlation analysis between theta activity measured during the encoding of picture context associations and subsequent spatial memory accuracy. In order to validate MEG effects found in healthy participants, we additionally acquired iEEG recordings while epilepsy surgery patients performed the task. We also had the exceptional opportunity of recording MEG and iEEG simultaneously in a patient with implanted depth electrodes in cortical regions of the left hemisphere. With this dataset, we were able to investigate how SMEs manifesting in local hippocampal activity were related to hippocampal cortical phase interactions.

\section{Materials and methods}

\subsection{Participants and data acquisition}

A sample of 13 healthy participants ( 8 females, mean \pm SD age: $23 \pm 3.7$ years; all right handed) were considered in the present study. MEG was recorded using a whole head system with 148 magnetometers (Magnes 2500 WH, 4D Neuroimaging, San Diego, CA, USA). Data were acquired at a sampling rate of $1017 \mathrm{~Hz}$ and on line band pass filtered between 0.1 and $400 \mathrm{~Hz}$. All participants had normal or corrected to normal vision and reported no history of neurological or psychiatric diseases.

Patients with drug resistant epilepsy were recruited over a period of 2 years, while undergoing iEEG monitoring at the University Hospital of Erlangen, Germany. A final sample of 12 patients ( 4 females, mean \pm SD age: $30.9 \pm 8.6$ years; all right handed) participated in the present study. These patients were implanted with a total of 866 iEEG contacts (diameter: $1.1 \mathrm{~mm}$; spacing: $4.5 / 5 \mathrm{~mm}$ ), distributed in 18 subdural (grids and strips) and 81 depth electrodes imbedded in silicon sheets (Fig. 1). Seven of the patients had depth electrodes targeting mediotemporal regions, containing contacts labeled as hippocampus (24) or entorhinal cortex (7). Out of the total, 10 patients performed the task on a bedside laptop, while iEEG was continuously sampled at $1024 \mathrm{~Hz}$ and referenced to white matter. Two patients participated in a simultaneous MEG and iEEG recording. Unfortunately, the head of one patient could not be positioned close to the MEG sensors, and only the iEEG data was analyzed. MEG was recorded using a whole head system with 248 magnetometers (Magnes 3600 WH, 4D Neuroimaging, San Diego, CA) housed in a magnetically shielded room at the same University Hospital. Data were acquired at a sampling rate of $2034 \mathrm{~Hz}$ and iEEG were average referenced. A successful simulta neous recording was obtained from a 43 year old patient (right handed female) implanted with 9 platinum strip electrodes. Contacts targeted lateral and mesial structures from parietal, temporal and frontal lobes of the left hemisphere. This patient suffered from a left temporo basal tumor (astrocytoma WHO grade II) that was completely resected 30 years before the iEEG monitoring. Presumably, the epileptic seizures were originated from the border of the resection. The clinical invasive recording revealed that seizure onset was medial to the resected region, detected by two electrode contacts (MNI coordinates [ $-34-17-30]$ and $[-39-17-29])$. All healthy participants and patients gave in formed consent before the experiment and performed the task in seated position.

\subsection{Task and stimuli}

The spatial navigation task was implemented as a video game using Unity3D (www.unity3d.com). The virtual environment depicted a cir cular field of grass surrounded by a wall, with distal cues consisting of mountains and a sky dotted with clouds. To navigate through this field, participants operated thumb controlled devices: either a joystick compatible with MEG (Celeritas Fiber Optic Response System) or a gamepad for recordings in the ward. Participants were instructed to ob serve pictures of buildings (shop facades and houses) at different loca tions within this field and remember the location in which each picture appeared. Each run of the task consisted of an encoding phase and a memory test (Fig. 2). An encoding trial started with a 3 s fixation period, after which the participant's point of view was situated at the center of the field, facing the sun (starting point). Participants had to find a 3D house model cueing a particular location and navigate to it. Navigation was divided in two steps: first, left and right turns were en abled until facing the cue; then, only forward (radial) movements. At the location, a picture was presented superimposed on the environment for $3 \mathrm{~s}$. The trial ended with a $1.5 \mathrm{~s}$ period where blinks were allowed. At test, each studied picture was presented on a black background after a fixation period. Participants were immediately placed in the field, at 

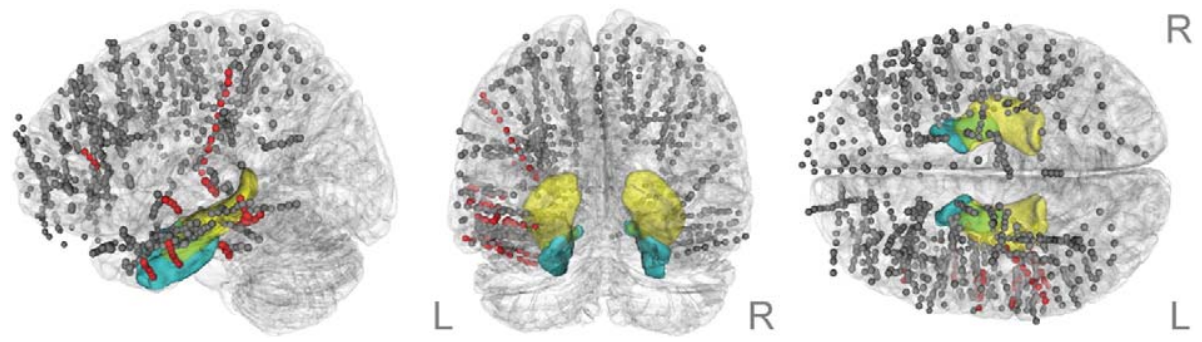

Fig. 1. Localization of all subdural and depth intracranial contacts (gray dots) in the MNI glass-brain representation ( $n=12$ patients). Contacts from the patient that underwent the simultaneous recording are highlighted in red. The hippocampal-entorhinal cortex complex is shown in color.

the starting point, and had to quickly point to the direction and move to the location where the picture was seen. Between encoding and retriev al phases, participants performed an interruption task consisting on counting backwards by 3 , starting from random numbers presented on the screen. Participants were reminded not to move their heads dur ing the recordings. The task was divided into blocks of about $18 \mathrm{~min}$ each. As a first control for ocular artifacts, we instructed the participants to refrain from blinking during fixation or picture periods.

A total of 36 pictures of real shop facades were collected from Wikimedia Commons (http://commons.wikimedia.org), preprocessed, and presented to healthy participants. For patients, we presented 36 pictures selected from the pool of images "buildings with gray back ground" used by (Bastin et al., 2013). Each picture was presented 3

\section{A}

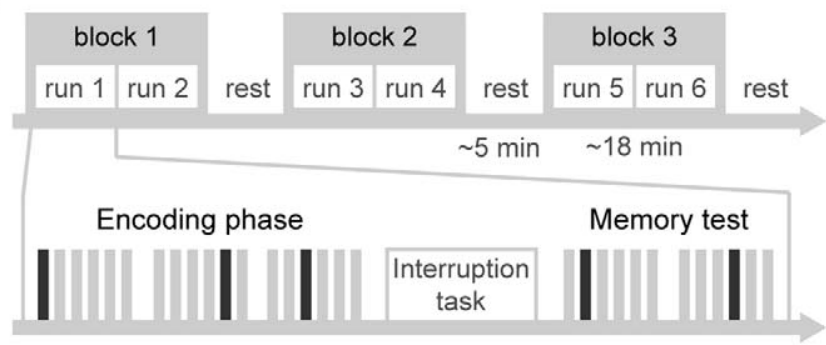

B

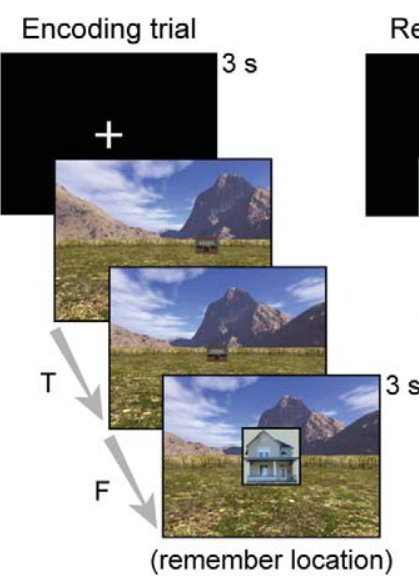

Retrieval trial

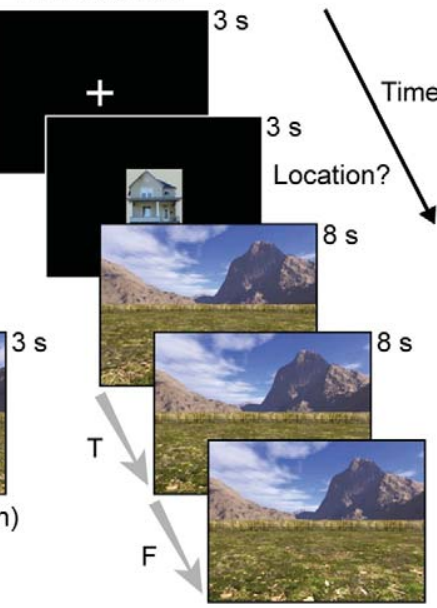

Fig. 2. Experimental design. (A) Block design (upper panel) and sequence of trials in a run of the subsequent memory task (lower panel). One of the trials (vertical bars) is highlighted to indicate that a particular picture is presented 3 times in the encoding phase and 2 times in the memory test, in a random order. (B) Trial structure of encoding and memory test. Gray arrows signal navigation periods when only left/right turns ( $\mathrm{T}$ ) or forward radial movements (F) are allowed (see Task and stimuli in Methods section for details). times at the same location during the encoding phase of the same run, and each location was associated with only one picture in all partic ipants. Each run normally included 6 different pictures, but in patients who exhibited relatively poor performance during training $(n=8)$, only 4 different pictures were used.

\subsection{Behavioral measures}

Participants' responses given in the memory test were registered in a $\log$ file. Spatial information was defined relative to the origin in the center of the field, where 'location' is represented by $(\mathrm{x}, \mathrm{y})$ coordinates in the horizontal plane and 'direction' is represented by the angle formed between the location and the $\mathrm{x}$ axis. A third spatial parameter 'radial distance' was defined as the Euclidean distance between the lo cation and the center of the field. Thus, we computed the errors of participant's responses for these three parameters. Location errors were computed as the Euclidean distance between the original location of the picture and the estimated location. Direction/radial distance er rors were computed as the absolute difference between the original di rection/radial distance of the picture and the estimated direction/radial distance, respectively. Direction errors were bounded between $0^{\circ}$ and $180^{\circ}$, that is, between zero and the maximum error, respectively. To ob tain a measure of spatial memory accuracy, location errors were multi plied by -1 before correlation analyses. Note that this transformation only changes the sign of the correlation but not its absolute value nor its statistical significance.

\subsection{MEG data preprocessing}

SQUID jumps, movement artifacts, strong muscle activity and blinks were visually identified on reference and MEG channels. All marked segments but blinks were data padded previous to independent com ponent analysis (ICA) to avoid discontinuities that could impair the al gorithm. To reduce environmental noise, we applied a regression method based on a subset of reference channels using the $\mathrm{ft} \_$denoise_pca function available in Fieldtrip toolbox (Oostenveld et al., 2011). To remove physiological noise (blinks, saccades, heart beats and muscle artifacts of small intensity) we applied the runica ICA algorithm (Bell and Sejnowski, 1995). These components were visu ally selected on the base of their characteristic time courses or/and fron tal/temporal topographic amplitude distributions. After data were segmented in the epochs of interest (see next section), trials were rejected if they contained segments initially marked as jumps, move ments, strong muscle activity or blinks. For source analyses, artifacts that may have remained in the data were expected to be attenuated by the beamformer, as shown by (Cheyne et al., 2007; Litvak et al., 2010). All healthy participants were presented with 108 trials of each epoch type during the encoding session; after initial trial rejection, a mean (SD) of 98 (9.8) trials were analyzed for each participant. 


\subsection{Time frequency analyses at sensor level}

Epochs were defined from -1 to $3 \mathrm{~s}$ relative to picture onset and from 0 to $3 \mathrm{~s}$ after fixation cross onset. Time frequency power was ob tained by applying the Morlet wavelet transform ( 6 cycles width) in the time domain to those epochs belonging to the learning phase. To minimize edge effects and artifacts from the blink period, we used $2.5 \mathrm{~s}$ mirror data padding on both sides of each epoch which were cut off after time frequency transformation.

Although our study is focused on the slow theta band we first per formed an analysis on the broad spectrum to facilitate comparison of SMEs with other studies and with other frequency bands. For this pur pose, power values were computed for 53 logarithmically sampled fre quencies between 1.5 and $140 \mathrm{~Hz}$ and for every time point. Power temporal resolution was down sampled by moving a $100 \mathrm{~ms}$ average window in steps of $50 \mathrm{~ms}$, which generated 80 time windows in the pic ture period. Fixation period was used as control condition, and a single mean value was considered. Power values were $\log$ and $z$ transformed according to the mean and standard deviation of the power across all picture and fixation cross events, computed separately for each time point, electrode and frequency bin. Each picture seen in the learning phase had associated location errors obtained later at test. To determine which part of the picture related activity showed SMEs, we performed correlation analyses following a hierarchical approach. First, we com puted Spearman's rank correlations between $z$ scored power values and location errors at subject level. Rho values were transformed to $t$ values generating individual time frequency $t$ statistical maps which were used for group level analyses. Participants' $t$ statistics were com pared against zero using the nonparametric Wilcoxon signed rank test, resulting in a single across participant $Z$ statistic for each sensor, time window and frequency. $P$ values were corrected for multiple com parisons across sensors and time frequency windows using the maxi mum statistic approach. An absolute significant threshold was set at the 97.5th percentile of a distribution obtained with 1000 Monte Carlo randomizations $(P<0.05$, two tailed). With each randomization, data and zero values were permuted in a random set of participants be fore applying the signed rank test.

Once we determined that slow theta band showed SMEs, we con ducted a second group level statistic in order to identify time frequency windows that were more suitable for source localization. This analysis was focused on a narrower frequency range including slow and classic theta bands $(1.510 \mathrm{~Hz})$. Firstly, we performed a signed rank test over 300 ms sliding windows for each frequency bin. Significance was determined by 1000 Monte Carlo randomiza tions and cluster maximum sum statistic across sensors $(P<0.05$, two tailed). In a second step, we visually compared statistical topo graphic maps of consecutive time windows within the largest signif icant area. If similar, these time windows were concatenated and subjected to another cluster based signed rank test with 1000 Monte Carlo randomizations ( $P<0.05$, two tailed). This step tested whether the effects in the selected time frequency window showed a consistent topography that could favor source localization.

\subsection{Source localization analyses}

Recent studies using simulations (Quraan et al., 2011) or a realistic anatomical and electrophysiological model (Attal and Schwartz, 2013) have provided evidence that MEG can detect hippocampal activity when it is combined with certain source localization techniques. In our study, we opted for source localization based on a vector linearly constrained minimum variance (LCMV) beamformer (Van Veen et al., 1997). First, MEG data were segmented in $3 \mathrm{~s}$ windows after fixation cross and picture onsets. The fixation period was used as a control condition, which is recommended for detecting weak sources in the presence of strong background noise (Quraan et al., 2011). Epochs were extended $2.5 \mathrm{~s}$ on both sides to prevent edge effects. Data were bandpass filtered in the frequency range of inter est using zero phase Kaiser windowed sinc FIR filters $(-6 \mathrm{~dB}$ cutoff at previously defined frequencies, transition width $0.5 \mathrm{~Hz}$, passband deviation of $0.1 \%$ and stopband attenuation of $-60 \mathrm{~dB}$, order 3624 ). The beamformer computation was based on the mean across trial covariance matrix of the time window of interest in picture and fixation epochs. The regularization parameter was set as $0.001 \%$ of the largest eigenvalue of the covariance matrix. Whole head power values were estimated as the sum of the 3 orthogonal power values obtained after spatially filtering the covariance matrix at each source location. Finally, event related synchronization (ERS; Pfurtscheller, 1992) values were computed by combining power from picture and fixation time windows.

Similarly to the time frequency analysis described above, we follow ed a hierarchical statistical approach. First, we computed Spearman's rank correlations between ERS values and position errors at the subject level. Then, rho values were transformed to $t$ values. Obtained individ ual source $t$ statistical maps were used for group level analyses. Partic ipants' $t$ statistics were compared against zero using the nonparametric Wilcoxon signed rank test, resulting in a single across participant $Z$ statistic for each voxel. Group level statistics were corrected for multi ple comparisons across voxels by applying false discovery rate (FDR) correction. Peak voxels from significant regions were labeled with xjView (http://www.alivelearn.net/xjview) which uses the WFU PickAtlas database (Maldjian et al., 2003).

\subsection{Time frequency analyses at source level}

Time frequency analyses at source level were focused on the hippocampal formation. To define this region of interest (ROI), we used probabilistic maps based on cytoarchitectonic delimitations derived from 10 post mortem human brains warped to the MNI template brain (Amunts et al., 2005). These maps contain the rela tive frequency with which a cerebral structure was present on each voxel of the anatomical MNI space. The source space was limited to the region having a probability of 0.1 or higher for hippocampal (CA, DG, Subc) and entorhinal cortex (EC) structures. We defined 277 source points within the ROI, using $5 \mathrm{~mm}$ spacing. For each source location, 3 time courses were extracted by projecting band pass filtered data through the orthogonal beamformer filters. Singu lar value decomposition was applied and the signal corresponding to the largest singular value was selected. This step was performed twice, for data filtered in the slow and classic theta frequency range. To inspect the extension of hippocampal effects and identify additional regions involved, we also performed a whole brain analy sis. Time frequency transformations were obtained in the same way as described for MEG channels, but restricted to each theta band. The resulting time frequency transformations were concatenated along the frequency dimension before statistical analyses. Correlation analyses were performed as previously described for MEG channels. $P$ values were corrected for multiple comparisons across time frequency bins and sources using FDR.

\subsection{Computation of the individual BEM head model}

The accuracy of forward models is very likely a significant factor in the detection of weaker sources, including deeper structures (Dalal et al., 2014). Since our study had a special focus on hippocam pal activity, we decided to create realistic 4 shell boundary element models (BEMs) based on anatomical information extracted from individual MRIs. Participants' T1 images were bias corrected, co registered to MNI space and resliced to $1 \mathrm{~mm}$ voxel size with SPM8. Resliced MRI images were automatically segmented by apply ing the method described in (Huang et al., 2013), which is based on the algorithm implemented in SPM8's "New Segment" toolbox (Ashburner and Friston, 2005). This segmentation is combined 
with an improved tissue probability map (TPM) developed at the Center for Advanced Brain Imaging at Georgia State University. The new TPM contains six tissue types (gray matter; white matter including the brain stem; cerebrospinal fluid [CSF] including inside ventricles and eyeballs; skull; scalp including soft tissues; and air) and it has an extended field of view that includes the whole head and the neck. Resulting tissue probabilities are smoothed and binarized. To fill CSF discontinuities, all gray matter voxels adjacent within $3 \mathrm{~mm}$ distance to the skull are reinterpreted as CSF (for more details, see procedure and code provided by Huang et al., 2013). The output consisted of tissues binary masks, which were modified to be compatible with BEM computation. The brain mask was derived from gray and white matter masks. Eyeballs were removed from CSF and reassigned to scalp in order to obtain closed and non intersecting layers. Scalp and brain masks were used to create respective boundary meshes in Fieldtrip which were smoothed afterwards. Skull and CSF (inner skull) were directly segmented and meshed in Fieldtrip. Meshes were built with approx imately 3000 vertices each. Tissue conductivities were set to 0.33 , $0.0041,1.79$ and $0.33 \mathrm{~S} / \mathrm{m}$, for scalp, skull, CSF and brain, respective ly. OpenMEEG (Gramfort et al., 2010; http://openmeeg.github.io/), was used to compute a BEM based forward model. To facilitate comparisons of equivalent brain sources across participants, source locations were defined in MNI coordinates using a $5 \mathrm{~mm}$ grid covering the brain. Then, individual MRIs were normalized to the standard T1 template provided with SPM8, using non linear trans formations. The inverse of this transformation was then applied to the template source grid with Fieldtrip, so that source reconstruction was performed in each individual's own brain space, but with consis tent MNI voxel locations across participants. MEG sensor coordinates were coregistered to each subject's MRI with the NUTMEG toolbox (Dalal et al., 2011) using fiducial and headshape points obtained with a Polhemus Fastrak digitizer (Colchester, VT, USA).

\subsection{Intracranial EEG data processing}

The MNI coordinates of iEEG contacts were determined by an expert neurologist based on postimplantation MRI and CT images, which were either normalized with SPM or processed with Curry (http://www. neuroscan.com). Once in MNI space, iEEG contacts were labeled with xjView (http://www.alivelearn.net/xjview) which uses WFU PickAtlas database (Maldjian et al., 2003). Contacts were visually inspected on postimplantation MRIs, and were classified as "white matter" only when they were at least $1 \mathrm{~cm}$ apart from gray matter, approximately. Contacts showing epileptogenic activity during stimulation or laying in lesioned regions were identified by expert physicians of the hospital. On this basis, signals were visually inspected before analyses to detect channels showing noise or substantial epileptic activity. Electrode chan nels laying in white matter or lesions or showing strong line noise arti facts, flat signals, or irregular activity were removed from analyses. Data was segmented in picture and fixation epochs. To detect spikes that may have remained in the data, we used a kurtosis criterion and excluded all segmented signals with kurtosis values higher than 4 . Kurtosis is a mea sure of the "peakedness" of a statistical distribution, and increases in the presence of outliers. We assumed that spikes will be detected as outliers because they appear in higher amplitude and less frequency than the background activity (Akiyama et al., 2012). After this procedure, we could analyze for each patient a mean (SD) of 37.0 (13.7) trials for each epoch type of the encoding session from a mean (SD) total of 51 (24.1) trials.

Power analyses were applied to iEEG data converted to the average reference. We chose this referencing scheme because it has been report ed to enhance theta signals (Zhang and Jacobs, 2015), probably imply ing less signal cancellation than bipolar schemes (i.e., no subtraction of adjacent neighbour signals). However, we performed supplementary analyses with the bipolar montage (each electrode was referenced to its neighboring electrode) for comparison with previous studies where this system has been extensively used. iEEG data were segmented in $3 \mathrm{~s}$ windows after fixation cross and picture onsets and time frequency analyses were performed following the same procedure as described for MEG channels but restricted to theta frequency band. To balance the number of trials across participants and electrodes, we set a fixed number of trials based on the minimum number of trials available for the regions of interest. Statistics were computed using 1000 random samplings of the data without replacement, by selecting 26 random tri als on each permutation. Significant correlations were corrected for multiple comparisons across time frequency windows, electrodes, and patients using the maximum statistic approach $(P<0.05$, two tailed).

For connectivity analyses, iEEG data were converted to the closest white matter reference to prevent spurious results caused by signal cancellation or volume conduction affecting other reference schemes (Arnulfo et al., 2015). The selection of the closest white matter elec trode was based on our own tissue classification and the proximity was decided after computing the Euclidean distances between MNI electrode coordinates.

\subsection{Phase coupling analyses between $I E E G$ and $M E G$ beamforming} sources

To estimate single trial event related functional connectivity be tween hippocampal slow theta sources and cortical activity simulta neously measured with iEEG and MEG, we chose the directed phase lag index or dPLI (Stam and van Straaten, 2012). This measure has been effective at identifying patterns of phase leading and lagging on simulated EEG data obtained from a realistic model of macroscopic structural and functional brain networks (Stam and van Straaten, 2012) and on real EEG data (van Straaten et al., 2015). Like the original PLI (Stam et al., 2007), the dPLI quantifies the phase synchronization strength between two signals, disregarding zero lag phase differences that may be due to volume conduction and/or common sources. This property is particular relevant for the assessment of functional connec tivity in presence of possible common MEG artifacts introduced by the intracranial electrodes during the simultaneous recording. However, the dPLI is distinctively computed by first applying the Heaviside step function to the phase differences, which assigns 0 to negative phase dif ferences, 1 to positive phase differences and 0.5 to zero phase differ ences. The final time averaged $\mathrm{dPLI}$ is also bounded between 0 and 1 , but it gives information of whether the reference signal is predominant ly leading $(0.5<\mathrm{dPLI} \leq 1)$ or lagging $(0 \leq \mathrm{dPLI}<0.5)$ the phase of the tar get signal. Note that in this case, both 0 and 1 indicate the strongest connectivity boundaries, whereas 0.5 marks the sign shift of the phase relations. By computing single trial dPLI between homologous signal pairs from two brain regions (source1 source 2 and iEEG2 source1) we can investigate whether the strength and the direction of the functional connectivity predicts memory performance. The latter information is used to compare the results and make apparent the sign flip of the ef fects (opposite correlation slopes) when exchanging reference and tar get regions.

For each voxel in the brain space, a slow theta source time course was reconstructed as described previously. Instantaneous phases of iEEG and source signals were obtained with the Hilbert transform. For each iEEG source or source source pair in a trial, we computed a single dPLI value resulting from averaging all dPLIs across the time window of interest. Next, we determined which dPLIs likely had a physiological meaning by means of surrogate tests that defined dPLI thresholds well above noise level. First, we split trials regarding the median value of as sociated behavior scores and computed mean dPLIs for two conditions (i.e., good and bad subsequent spatial accuracy) for each reference target signal pair. Then, we generated 200 surrogate dPLIs by applying a random temporal shift to the phase time series of the target signal within the analyzed epoch. Surrogate dPLI values were computed fol lowing the same procedure described above for computing dPLIs in 
the non shifted data. dPLI significance thresholds were set to the 95th quantile of each surrogate dPLI distribution $(P<0.05)$. If mean dPLIs in at least one of the two conditions exceeded the surrogate threshold, the following analyses were additionally applied: Spearman's rank cor relations with subsequent spatial accuracy and Wilcoxon rank sum tests were applied on single trial dPLI source maps. $P$ values were corrected using cluster based permutation tests (1000 Monte Carlo randomizations). To minimize type I error caused by multiple tests, a single threshold $(P<0.05)$ was obtained from the distribution of maxi mal sum cluster statistics derived from all correlations or unpaired tests. To analyze in more detail possible zero lags between sources, we generated 1000 surrogate phase differences using the described temporal shift method and computed the summed probability of phase differences in 16 angle bins.

\section{Results}

Our goal was to investigate whether human hippocampal slow theta activity is involved in the formation of accurate contextual spatial locations supporting navigation and to determine the sign of this rela tionship. For this purpose, we applied correlation analyses between slow theta activity time locked to the encoding of item place associa tions and subsequent spatial accuracy for contextual locations. This analysis was performed on MEG and iEEG data collected from 13 healthy participants and 12 neurosurgical patients, respectively. One of these patients was recorded with iEEG and MEG simultaneously, allowing us to investigate the phase relations between hippocampal slow theta sources showing local SMEs and cortical regions traditional ly associated with successful encoding.

\subsection{Behavioral results}

Healthy participants made mean $( \pm S D)$ errors for location, direction and radial distance of $15.8 \pm 6.0$ virtual meters $(\mathrm{vm})$, $16.8^{\circ} \pm 11.6^{\circ}$ and $10.5 \pm 2.0$ vm respectively. Neurosurgical patients made significantly larger mean errors for all measures (location: $40.0 \pm 18.8 \mathrm{vm}$, Mann Whitney $U=108, P=0.001$; direction: $45.2^{\circ} \pm 26.0^{\circ}$, Mann Whitney $U=120, P=0.008$; radial distance: $17.2 \pm 8.8 \mathrm{vm}$, Mann Whitney $U=114, P=0.003)$. The patient WM, who participated in the simultaneous recording, made large di rection errors $\left(75.0^{\circ} \pm 50.6^{\circ}\right)$ that differed significantly from the pa tients' mean (one sample Wilcoxon signed rank $=2, P=0.003$ ). However, the mean error for location $(50.9 \pm 19.1 \mathrm{vm})$ remained similar to the rest of the patients $(P>0.17)$, likely due to the small error committed in the estimation of the radial distance (12.9 \pm $10 \mathrm{vm})$.

\subsection{Slow theta power decreases after stimulus presentation are beneficial} for accurate encoding of the contextual location

To determine which part of the sensor level oscillatory activity showed subsequent memory effects in healthy participants, we per formed correlation analyses between time frequency power of picture periods in the learning phase and participants' spatial accuracy obtained in the context memory test. A first analysis performed on the broad spectrum $(1.5 \mathrm{~Hz}$ to $140 \mathrm{~Hz})$ showed main post stimulus SMEs in slow theta $(25 \mathrm{~Hz})$ and beta, gamma and high gamma oscillatory ac tivity (Supplementary Fig. 1; $P<0.05$, corrected with randomizations and maximum statistic; all absolute Wilcoxon signed $\operatorname{rank} Z>2.67$; $n=13$ subjects). In order to identify time windows that were more suitable for source localization of slow theta SMEs, across sensor cluster analyses were performed in the $1.510 \mathrm{~Hz}$ frequency range. The two step sliding window analysis identified a cluster showing consistent to pographic effect, comprising frontal, temporal and posterior sensors of the right hemisphere $(P$ cluster $<0.001$; all Wilcoxon signed rank $Z<-1.71$; Fig. 3A,B). This cluster indicated that there was a significant
A

B
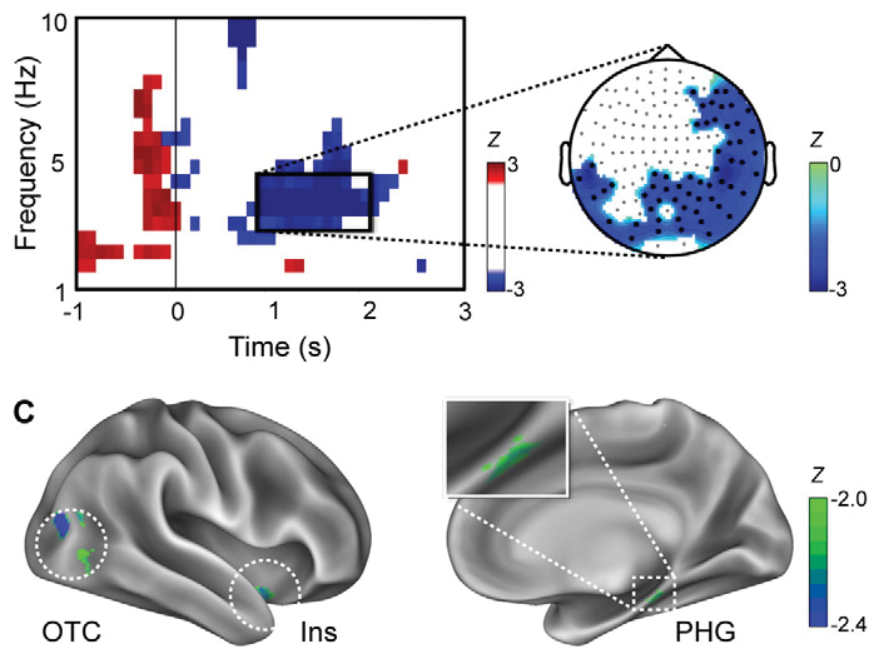

D
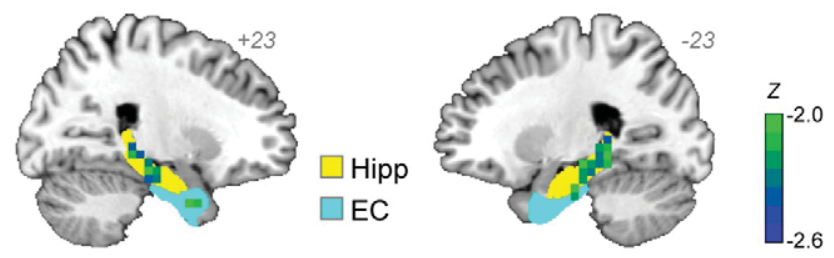

E
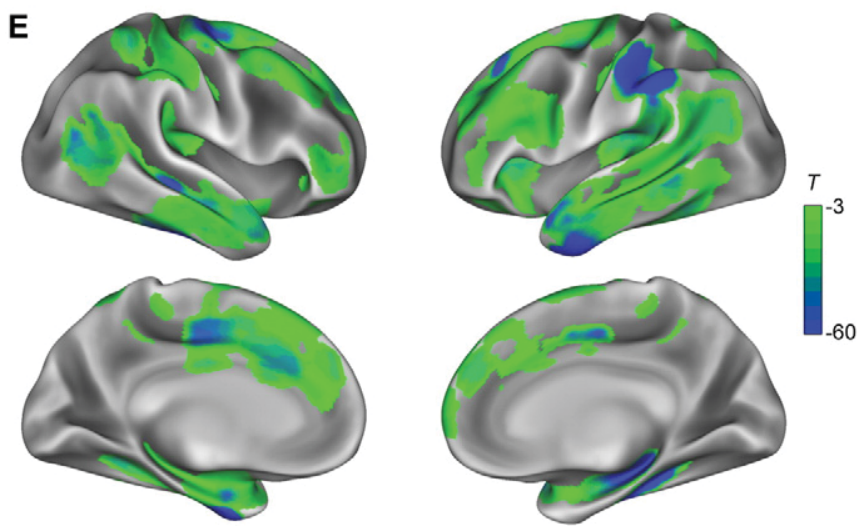

Fig. 3. Power analyses showing negative correlation between theta oscillatory activity and subsequent spatial accuracy. (A) Time-frequency representation showing the results from the two-step sliding-window analysis applied to MEG data recorded from healthy participants $(n=13)$. The black frame encloses the negative cluster surviving the second step (P-cluster $<0.001 ; 0.9-2.21 \mathrm{~s}, 3-4.5 \mathrm{~Hz})$. (B) Topographic plot of the negative cluster showing mean Wilcoxon signed-rank $Z$ across time-frequency bins included in the significant window. Highlighted black dots represent sensors that belonged to the cluster at any time-frequency bin. (C) Source localization of the negative cluster detected at sensor level, projected to the surface of the inflated brain representation ( $P<0.05$, FDR corrected). (D) Results from the source-level timefrequency analysis performed within the ROI, depicted in sagittal slices of both brain hemispheres. Green-to-blue small squares represent significant voxels $(P<0.05$, FDR corrected) in hippocampus (Hipp, yellow mask) and entorhinal cortex (EC, cyan mask). (E) Results from the time-frequency correlation analysis applied to intracranial averagereferenced EEG data, projected to the surface of the inflated brain representation. Greento-blue areas represent mean $t$ statistics across all contacts included in $12.5-\mathrm{mm}$ spherical voxels. The $t$ statistic of each contact is defined as the sum of all significant $t$ values across time-frequency bins $(P<0.05$, corrected with randomizations).

negative correlation between slow theta power ( $34.5 \mathrm{~Hz}$ ) and subse quent spatial accuracy between $0.92 .21 \mathrm{~s}$ after picture onset (Fig. 3B). This effect remained when only including a subset of trials defined as 'hits' for the background context (Supplementary Fig. 2). The whole brain analysis revealed the main beamforming sources in the right 
occipitotemporal cortex (middle occipital gyrus: MNI coordinates [45 -80 10], BA $19, Z=-2.83$; middle temporal gyrus: MNI coordinates [45 - 75 10], BA 39, $Z=-2.55$ ), right insular cortex (MNI coordinates [40 $5-10$ ], BA 13, $Z=-2.69$ ), right MTL (hippocampus: MNI coordi nates [20 - $30-10$ ], BA 28; parahippocampal gyrus: MNI coordinates [25 - 30 - 15], BA 36; both $Z=-2.06$ ) and right cerebellum (culmen: MNI coordinates [15 $-35-15], Z=-2.76$; all $P<0.05$, FDR corrected; all Wilcoxon signed rank $Z<-1.99$; Fig. 3C). A further bootstrapping analysis with replacement showed that source effects were robust $(<-2 \mathrm{SD})$ and not specific to the particular sample. The two step sliding window analysis also showed positive correlations between theta activ ity $(38 \mathrm{~Hz})$ and spatial accuracy in the pre stimulus interval $(P$ cluster $<0.001$; all Wilcoxon signed $\operatorname{rank} Z>1.57$ ) but it was not possi ble to localize the sources of this effect, probably due to its short duration.

The main objective of our study was based on a strong a priori knowledge about the role of the hippocampal entorhinal complex in the processes evaluated here (i.e., spatial coding and episodic memory formation), as well as the functional link between this structure and the generation of slow theta oscillations. To enhance the detection of hippocampal activity, the lead field was computed at equivalent anatomical positions across participants and a hippocampal entorhinal cortex region of interest (ROI) was defined based on tissue probabilistic maps (see Section 2.7 for details). Then, we performed a time frequency correlation analysis focused on the sources comprised within this ROI. This analysis showed that also slow theta activity in mid posterior hippocampi and entorhinal cortices was negatively correlated with subsequent spatial accuracy $(P<0.05$, FDR corrected; all Wilcoxon signed rank $Z<-2.18$; Fig. 3D), although effects were evident in slower frequency bins $(\sim 23 \mathrm{~Hz})$ and a later time window ( $1.52 .5 \mathrm{~s}$; Supplementary Fig. 3). The whole brain analysis revealed other important negative effects in right amygdala, as well as retrosplenial and cingulate cortices, thalamus and basal ganglia $(P<0.05$, FDR corrected; all Wilcoxon signed $\operatorname{rank} Z<-2.03)$. Note that although stimuli elicited theta power increase relative to baseline (fixation period) at sensor and source level (Supplementary Fig. 4A), our results are in line with abovementioned reports of power decreases for successful encoding relative to unsuccessful encoding (Supplemen tary Fig. 4B).

In order to validate results found with MEG data, we applied time frequency correlation analyses to intracranial EEG recorded from neu rosurgical patients. We observed that theta oscillatory power exhibited both positive and negative correlation with subsequent spatial accuracy in widespread regions $(P<0.05$, corrected with randomizations and maximum statistic; all absolute $t>2.31$ ). We focused on contacts (including 11 labeled as hippocampus) showing negative SMEs. As Fig. 3E shows, the effects overlapped with results obtained in healthy participants over right occipito temporal cortex and right mid posterior hippocampus. The effects were similarly distributed when using a bipolar montage (Supplementary Fig. 5), although there was an attenuation over some areas probably due to signal cancellation. However, we did not observe statistical patterns that were specific of a particular frequency band or temporal window.

3.3. Phase coupling between MEG sources and simultaneously recorded cortical activity during slow theta negative SMEs

It has been proposed that power decreases in low frequency activity during episodic memory formation reflects reduced local correlation between neuronal firing rates and allows increased information pro cessing across a large network (Greenberg et al., 2015). Such neural desynchronization could induce synaptic plasticity (Staudigl and Hanslmayr, 2013), a mechanism dependent on the phase of hippocam pal oscillations (Pavlides et al., 1988, p. 198). We investigated this hy pothesis by testing whether memory related decreases in slow theta activity were associated with changes in long range functional connectivity. This analysis was performed in the simultaneous MEG iEEG dataset to be able to compare results based on source data alone to those obtained when including intracranial activity. Fig. 4 summa rizes the results of this single subject analysis. First, we determined whether hippocampal activity after picture onset was correlated with subsequent spatial accuracy by performing a time frequency source analysis within the defined ROI. Like in healthy participants, the cluster based permutation test revealed a significant negative correla tion between slow theta activity $(\sim 23 \mathrm{~Hz})$ and subsequent spatial ac curacy $(P$ cluster $=0.038$; all $t<-2.03$, corresponding to Spearman's $R<-0.33 ; n=36$ trials; Fig. 4B). As depicted in Fig. 4A,C, the effect was stronger in ROI's anterior voxels of the right hemisphere, at a late time window (1 $3 \mathrm{~s}$ ). Next, we estimated slow theta phase synchroni zation between the source showing maximal correlation in the right an terior hippocampus (MNI coordinates [20 - $10-20]$, BA 54; mean Spearman's $R=-0.56, P<0.001)$ and source activity from the rest of the brain space. We found a significant phase coupling between the right anterior hippocampus and the left lateral temporal lobe in trials associated with better subsequent performance, where the former re gion was phase leading the latter (peak coupling in middle temporal gyrus: MNI coordinates $[-65-35-10]$, BA 21, mean dPLI $=0.66$ higher than surrogate threshold, $P=0.035$; peak difference between conditions in inferior temporal gyrus: MNI coordinates $[-55-25$ -20 , BA 20, Wilcoxon rank sum $Z=3.24$; $P$ cluster $<0.01$, all $Z>2.13$ ). Furthermore, dPLI values were correlated with spatial accura cy for location indicating that the stronger the right anterior hippocam pus was phase leading, the better the subsequent performance (peak correlation in inferior temporal gyrus: MNI coordinates [ $-60-55$ -15 ], BA 20, Spearman's $R=0.66, P<0.001 ; P$ cluster $=0.01$; Fig. 4D). This result could be validated by performing a reverse analysis using temporal activity recorded intracranially (reference contact TD3: MNI coordinates $[-57-38-20]$, BA 20, Spearman's $R=-0.50$ $P=0.002 ; P$ cluster $=0.02$; Fig. $4 \mathrm{E}$; closest source to TD3: MNI coordi nates $\left[\begin{array}{lll}-60 & 40 & 20\end{array}\right]$, Spearman's $R=-0.42, P=0.01$ ).

Interestingly, intracranial activity in the left temporal cortex (refer ence contact TA5: MNI coordinates [ $-53-5-29]$, BA 21) also showed a significant coupling with the left inferior frontal gyrus (MNI coordi nates [ -50200 ], BA 47) that was modulated by performance (mean dPLI good $=0.39$, mean dPLI bad $=0.61$, Wilcoxon rank sum $Z=-3.34, P=0.03 ; P$ cluster $=0.03$ ). The stronger temporal activity was driven by the left inferior frontal gyrus (smaller dPLI values), the better the subsequent spatial memory (Spearman's $R=-0.61$, $P<0.001 ; P$ cluster $=0.01 ;$ Fig. $4 \mathrm{~F})$. This result could be validated either by using a close source as the reference (MNI coordinates $[-50-5$ $-25]$, BA 20; Spearman's $R=-0.53, P<0.001$ ) or by performing a re verse analysis using the left inferior frontal source as reference. Curious ly, the latter analysis revealed that the inferior frontal gyrus was targeting a similar area in the left temporal lobe as the right anterior hippocampus (peak correlation in inferior temporal gyrus: MNI coordi nates [ $-55-50-15]$, BA 20; Spearman's $R=0.65, P<0.001 ; P$ clus ter $=0.01$; Fig. $4 \mathrm{G})$.

The dPLI, by definition, protects against the possibility that connectivity effects may be due to instantaneous (zero lag) correla tions caused by mislocalization of MEG sources in two different locations. Supplementary Fig. 6A,B shows that phase differences between synchronized sources were not concentrated at zero $(P<0.05$, surrogate test $)$ and had a significant phase shift $(P<0.01$, surrogate test). The same does hold true for the hippocampal and frontal sources although they were coupled to overlapping temporal regions (Supplementary Fig. 6C).

\section{Discussion}

Based on several findings, it has been proposed that human hippocampal slow theta activity is analogous to the theta rhythm in rodents (Jacobs, 2014; Watrous et al., 2013) and may be positively 
correlated with memory formation (Lega et al., 2012). Here, we investigated the relationship between hippocampal slow theta activity and subsequent spatial memory accuracy in navigation. Although previous MEG studies evaluating subsequent navigation performance seem to support this hypothesis (Cornwell et al., 2008; Kaplan et al., 2012), slow theta activity related to the forma tion of item location associations during navigation has not been

A
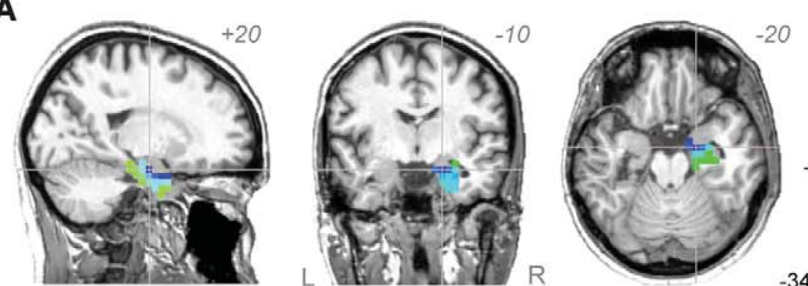

$T$

$-340$

B

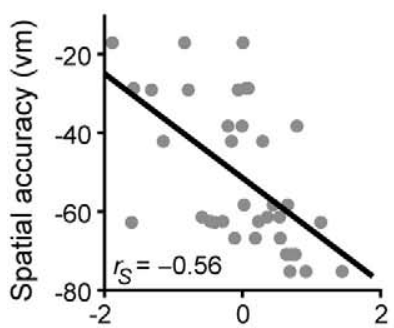

C

Slow-theta power (ERS)
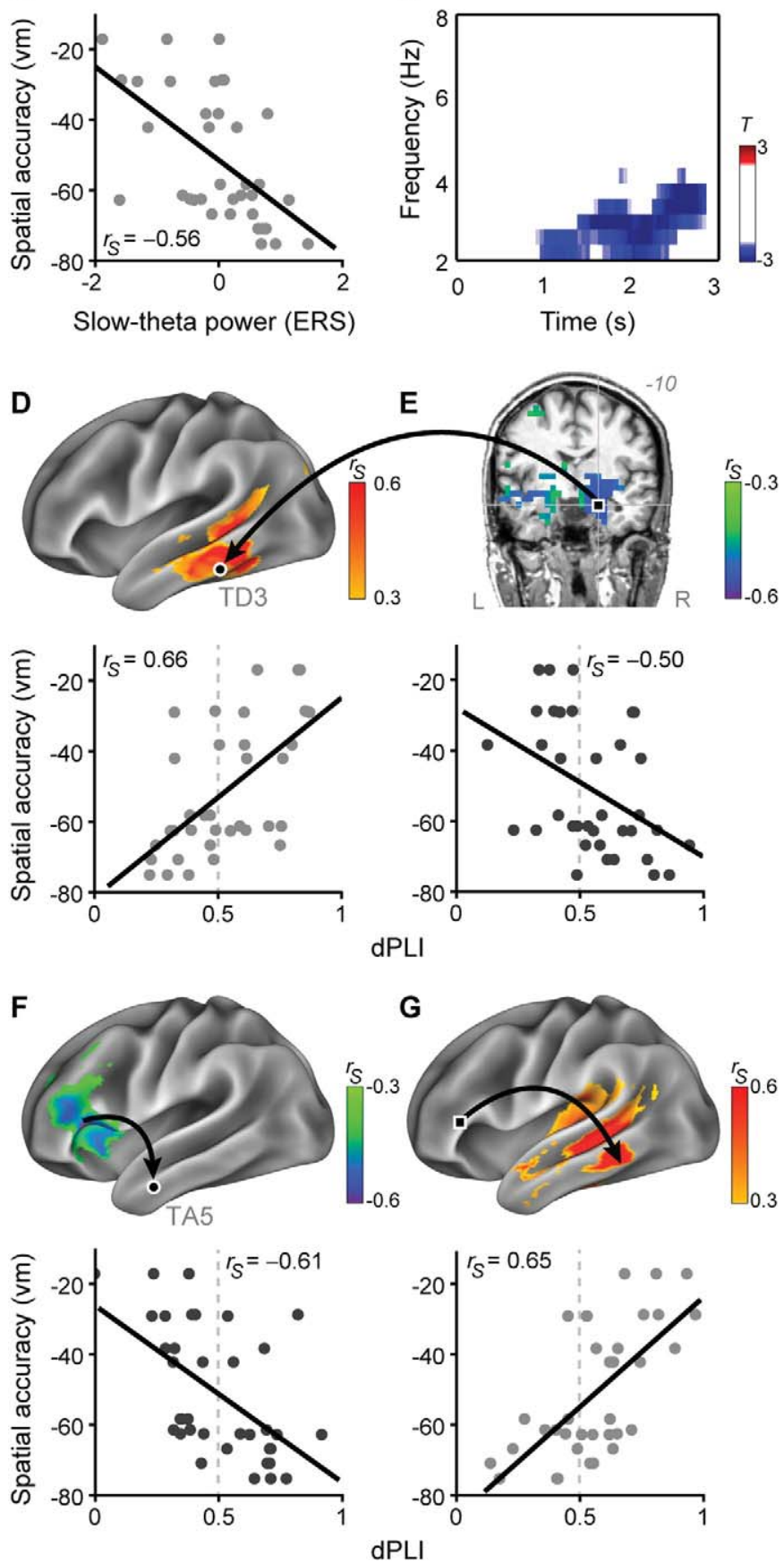

directly assessed until now. To make this possible, we administered the navigation task in the form of a subsequent memory paradigm where pictures of buildings were presented at particular spatial loca tions in a virtual grass field. This setting allowed us to analyze oscil latory activity time locked to stimulus onset during the encoding phase. Contrary to our working hypothesis, we found that hippocam pal slow theta activity and later spatial accuracy for the recalled locations were negatively correlated. Additional negative SMEs were localized in cortical and MTL regions of the right hemisphere, which were likely engaged in spatial processing. A brief positive SME was observed in prestimulus broadband theta at left frontal sensors, but it was not observed in hippocampal source analysis. A simultaneous MEG and intracranial EEG dataset in an epilepsy surgery patient showed that during power decreases, slow theta in right anterior hippocampus and left inferior frontal gyrus phase led the left lateral temporal cortex and predicted spatial accuracy. Our results indicate that accurate encoding of contextual spatial locations benefits from decreased slow theta activity in hippocam pus and task related regions. As discussed below, this effect may reflect local and long range neural mechanisms facilitating increased local processing of spatial information during the acquisition of item place associations.

\subsection{Sources of slow theta negative subsequent memory effects are found in task relevant regions}

We observed that slow theta negative SMEs were not widespread but focally localized in a set of MTL and cortical regions, strongly sug gesting that power modulations were correlates of task specific pro cessing. Part of these effects was apparent in the hippocampal entorhinal complex, a core system for memory and spatial navigation and functionally linked to theta oscillations (Buzsáki and Moser, 2013). Acknowledging the importance of this system for the successful performance of our study's task, by extension, it is plausible that slow theta negative SMEs manifested in actively engaged brain regions. In agreement, negative SMEs were significant in posterior hippocampi, which typically show increased BOLD activation during the encoding and retrieval of spatial memories, in contrast with anterior hippocampi

Fig. 4. Results from the single patient that underwent a simultaneous iEEG-MEG recording. (A) Cluster of voxels in the right mid-anterior hippocampus showing significant negative correlation between theta power and subsequent spatial accuracy. The color code indicates the sum of significant $t$ values across time-frequency bins. (B) Negative correlation between mean slow-theta power in right anterior hippocampus (voxel showing the maximal summed $t$ statistic in MNI coordinates [20 $10 \quad 20]$ ) and subsequent spatial accuracy. Power was averaged across significant time-frequency bins. (C) Time-frequency representation of the significant correlation found in the right anterior hippocampal source $(P$-cluster $=0.038$; $n=36$ trials). (D-G) Results from Spearman's correlation analysis applied to dPLIs computed between either a MEG source (D, G) or a intracranial EEG reference signal $(E, F)$ and the whole source space. Color areas represent target anatomic regions of significant dPLI interactions that either correlated positively (warm colors) or negatively (cold colors) with subsequent spatial accuracy. (D) Significant dPLI cluster between right anterior hippocampal source (reference) and sources in left inferior and middle temporal gyri $(P$-cluster $=0.01$ ). The arrow indicates that performance was better when the reference source was phaseleading. Lower panel shows the correlation plot found in the source showing the peak correlation (inferior temporal gyrus: MNI coordinates [ $60 \quad 55 \quad 15]$, BA 20; $P<0.001$ ). (E) Significant dPLI cluster between the reference iEEG contact TD3 and sources in right hippocampus ( $P$-cluster $=0.02$ ). The arrow indicates that performance was better when the intracranial signal was phase-lagging. Lower panel shows the correlation plot found in right anterior hippocampus (MNI coordinates $\left[\begin{array}{lll}20 & 10 & 20\end{array}\right]$, BA 20; $\left.P=0.002\right)$. (F) Significant IPLI cluster between the reference iEEG contact TA 5 and sources in left inferior frontal gyrus $(P$-cluster $=0.01)$. The arrow indicates that performance was better when the intracranial signal was phase-lagging. Lower panel shows the correlation plot found in the source showing the peak correlation (inferior frontal gyrus: MNI coordinates [ 5020 0], BA 47; $P<0.001$ ). (G) Significant dPLI cluster between left inferior frontal source (reference) and sources in left inferior and middle temporal gyri $(P$-cluster $=0.01)$. The arrow indicates that performance was better when the reference source was phase-leading. Lower panel shows the correlation plot found in the source showing the peak correlation (inferior temporal gyrus: MNI coordinates [ 5550 15], BA 20; $P<0.001) . \mathrm{r}_{\mathrm{S}}=$ Spearman's correlation coefficient. 
(Duarte et al., 2014; Nadel et al., 2013). As an fMRI study applying multivoxel pattern similarity analysis has demonstrated, right posterior hippocampus specifically carries information about spatial locations during the encoding of object location relationships in working memo ry (Libby et al., 2014). As validation of our MEG results, correlation anal yses performed in deep contacts of epileptic patients also showed theta negative SMEs mostly localized in right posterior hippocampus. Partic ularly salient is that the place cells of posterior hippocampus have the smallest place fields (Nadel et al., 2013), which suggests that this struc ture is critical for encoding locations with a fine grained spatial resolu tion. Additional negative SMEs found in entorhinal cortex (EC) could reflect the gating of visuospatial and visual object information coming from different cortical pathways to hippocampus. Moreover, it is known that entorhinal grid cells themselves encode spatial representa tions of the environment using information derived not only from path integration (Hafting et al., 2005), but also from visual exploration with out locomotion (Killian et al., 2012).

Negative slow theta SMEs were also localized in sources of occipitotemporal cortex, insular cortex and parahippocampal gyrus (PHG) of the right hemisphere. Correlation analyses performed on intra cranial data confirmed negative SMEs in right occipitotemporal cortex and revealed additional memory related modulations in the left hemi sphere, including inferior parietal gyrus. The involvements of PHG and occipitotemporal cortex are in line with fMRI findings revealing the im portance of these structures for topographical learning during naviga tion (Maguire, 1997). These regions contain the parahippocampal and occipital place areas, which play a role in coding the identity of land marks and its visual features, respectively (Marchette et al., 2015). Par ticularly, parahippocampal place area may have contributed to encode environmental scenes, but also the pictures of buildings presented at each spatial location (Bastin et al., 2013). Finally, although the role of in sular cortex is less clear, studies in rats have provided evidence that this structure is involved in memory formation, memory consolidation, ob ject recognition memory and spatial learning (Bermudez Rattoni, 2014). Interestingly, right insula and left inferior parietal gyrus are among the regions showing context dependent SMEs at $\sim 4 \mathrm{~Hz}$ theta frequency (Staudigl and Hanslmayr, 2013).

4.2. Slow theta power decreases may be a marker of increased neural activation

Assuming that the aforementioned regions actively encoded task relevant information, a straightforward interpretation is that slow theta power decreases may be a marker of increased neural activation. To un derstand how slow theta power decreases and memory encoding are mechanistically related, we follow a similar perspective previously of fered for explaining negative SMEs in low frequency activity (Greenberg et al., 2015; Staudigl and Hanslmayr, 2013). It is known that both sensory stimulation and top down attention cause cortical desynchronization, which is expressed as power reductions in local field potential and re duced correlation between neuronal firing rates (Harris and Thiele, 2011; Pfurtscheller and Aranibar, 1977). Greenberg and colleagues (Greenberg et al., 2015) have suggested that decreases in broad theta (3 $8 \mathrm{~Hz}$ ) activity during episodic encoding may simply reflect decreases in neuronal correlations across cortical and MTL regions, which make the network more efficient for storing information. Likewise, the closely related information via desynchronization hy pothesis also suggests that neural desynchronization is a prerequi site for information encoding (Hanslmayr et al., 2012). Although the traditional view is that synchronized oscillations support learning by facilitating synaptic links between neurons, Hanslmayr and colleagues (Hanslmayr et al., 2012) explain that long term po tentiation (LTP) can also benefit from desynchronized activity in other neuronal circuits. There is now convincing evidence of these views, revealed by a recent experiment evaluating associative encoding under a spatial mnemonic strategy (MC Fellner, personal communication, December 2015). The study demonstrates that theta power decreases co occurred and were co localized with BOLD signal increases in MTL, a region clearly driven by task's pro cesses. The same reasoning could be applied to decreases in slow theta $(\sim 25 \mathrm{~Hz})$ activity found in our study, taking also into account the high overlap in frequency with broadband negative SMEs (Burke et al., 2013; Greenberg et al., 2015; Long et al., 2014; Sederberg et al., 2007). In concordance with these two perspectives, decreased slow theta activity could mean that higher degree of visual and spatial in formation was processed and encoded into memory, enabling a more accurate retrieval of the spatial location during the test phase. Im portantly, our analysis can give more robust support for this inter pretation than a contrast between conditions (e.g., remembered vs. forgotten). Note that the negative SME reported here is the result of a correlation between slow theta power and spatial accuracy, which shows a statistical dependence between both variables across trials. The small mean location, direction and radial distance errors made by the healthy participants when indicating the remembered loca tions demonstrates that in most of the cases they were able to recall some contextual details; therefore, the spatial accuracy of their responses greatly depended on how detailed and specific their memory representa tions were encoded. On the other hand, the individual dataset of the simul taneous recording showed peak negative correlation in the right anterior hippocampus, a critical region for item context binding (Libby et al., 2014; Staresina and Davachi, 2009). Curiously, this patient was particularly less accurate than other participants in indicating the right direction where the picture was presented, which mostly depended on associating the pic tures to their right distal background contexts.

Despite this interpretation, our results may seem contradictory to those generated by a recent analysis of slow theta to gamma phase amplitude coupling (PAC) in human hippocampus (Lega et al., 2016). Lega and colleagues found a positive relationship between slow theta SMEs and PAC during the encoding of words. Furthermore, they found that the specific hippocampal electrodes exhibiting greater PAC during successful than unsuccessful encoding (PAC + ) showed a preferred phase at the trough of the slow theta oscillation, where LTP is expected to be facilitated. On the contrary, hippocampal PAC - electrodes (associated with negative SMEs) exhibited theta gamma coupling at phases more likely to induce long term depres sion (LTD). As discussed in Lega et al., 2016, slow theta power decreases might reflect the disengagement of hippocampus from the global slow activity; because only a small subset of locations would show the opposite pattern, power decreases are more likely to be non invasively detected. An exciting alternative is that slow theta negative SMEs found in our study reflect spatial learning facilitated by LTD. Indeed, accumulating evidence from rodent studies is showing that LTD is fundamental for the formation of declarative memories, and plays a distinctive role in the processing of object place contextual information (Kemp et al., 2013).

\subsection{Poststimulus slow theta power decreases were not associated with prestimulus theta power increases}

Together with slow theta negative SMEs, we additionally observed a transient positive correlation between prestimulus theta activity and spatial memory accuracy at left frontal sensors. Our results agree with a previous study showing that frontal theta activity before the onset of a retrieval cue predicts the subject's ability to remember episodic details (Addante et al., 2011). The authors of that study have proposed that prestimulus theta may reflect a cognitive brain state facilitating the pro cessing of the retrieval cue and following recollection. We could equally reason that enhanced theta activity facilitated the processing of the in coming picture and the recollection of spatial information (e.g., the path from the starting point to the current location), stored in the same or a previous trial during the encoding phase. Recollected spatial information could have been associated with the current event (picture 
at location), adding more details to the memory trace. Alternatively, poststimulus slow theta power decreases could be a consequence of prestimulus theta power increases. This possibility seems unlikely be cause both effects were visible in well separated brain regions. In fact, as we confirmed with post hoc analyses, we did not find positive prestimulus SMEs in hippocampal sources. Although other studies ana lyzing the encoding of words have found positive SMEs in prestimulus theta of MTL regions, including hippocampus (Fell et al., 2011; Guderian et al., 2009), it was recently demonstrated that increased prestimulus hippocampal theta activity only predicts subsequent item recognition (Merkow et al., 2014). However, free recall, which depends more on associative encoding as our task, shows a negative SME in post stimulus hippocampal slow theta.

\subsection{Successful reconstruction of slow theta sources in the hippocampus}

We discovered memory related modulations of $23 \mathrm{~Hz}$ power in MEG source reconstructions of the hippocampus, corresponding to the dominant oscillatory frequency of this structure in humans (Watrous et al., 2013). This is consistent with other MEG experiments evaluating memory and spatial navigation which have also reported hippocampal effects at slower theta frequencies (Cornwell et al., 2008; Kaplan et al., 2012; Staudigl and Hanslmayr, 2013). Although it has been suggested that the hippocampus may form a closed electric field due to its sym metrical morphology (Lorente de No, 1947), this would require that the entire structure be simultaneously activated, a rather unlikely sce nario under typical experimental conditions. Moreover, a closer look at hippocampal slices reveals asymmetrical sections and an uneven dis tribution of pyramidal neurons, further reducing its effective symmetry. Finally, the depth attenuation of neuromagnetic sources (Hillebrand and Barnes, 2002) may be partially offset by the much higher density of pyramidal cells (and corresponding current density) in hippocampus relative to other cortical tissue (Attal et al., 2012). Indeed, simultaneous MEG and intracranial data from drug resistant epileptic patients strong ly suggest that MEG can detect hippocampal theta activity. With such recordings, it has been observed that surface magnetic activity and hip pocampal theta recorded with depth electrodes were practically over lapped, without phase delay (Dalal et al., 2013). Another simultaneous MEG iEEG study demonstrated that epileptic spikes generated in the hippocampus are sometimes observable with MEG (Santiuste et al., 2008). In the present study, we showed that hippocampal cortical phase synchronization was similar using either cortical iEEG signals or equivalent beamforming sources.

\section{Conclusions}

In conclusion, our study demonstrates a negative relationship be tween the accuracy of spatial memories and MEG slow theta activity generated in hippocampus and cortex during the formation of item place associations. Negative SMEs in hippocampus are concomitant with more consistent phase coupling between hippocampus and key cortical regions for successful memory encoding. These phenomena suggest that decreased slow theta activity reflects underlying local and long range neural mechanisms facilitating the encoding of more ac curate spatial memories. Further studies are still necessary to clarify the specific role of different human theta bands observed across cortical and MTL regions, and how negative/positive SMEs may be related to the in duction of LTD/LTP in support of new memories.

\section{Acknowledgments}

This work was supported by the Zukunftskolleg of the University of Konstanz (to M.C.G. and S.S.D.); the Deutsche Forschungsgemeinschaft DFG (grant number DA $14851 / 1$ to S.S.D.) and ERA Net NEURON via the Bundesministerium für Bildung und Forschung BMBF (grant num ber $01 \mathrm{EW} 1307$ to S.S.D.).
We are very grateful to Marie Christine Fellner for her helpful com ments on the manuscript, Daniel Wong for implementation of the BEM pipeline and Julien Bastin for kindly providing the images of buildings used as stimuli in this study. We kindly thank Mathis Kaiser, Ursula Lommen and Barbara Awiszus for their help during the MEG recordings and Martina Rzonsa for her help during the simultaneous iEEG MEG re cording. We would like to give special thanks to the patients and other volunteers for taking part in this study.

\section{Appendix A. Supplementary data}

Supplementary data to this article can be found online at http://dx. doi.org/10.1016/j.neuroimage.2016.08.021.

\section{References}

Addante, R.J., Watrous, A.J., Yonelinas, A.P., Ekstrom, A.D., Ranganath, C., 2011. Prestimulus theta activity predicts correct source memory retrieval. Proc. Natl. Acad. Sci. U. S. A 108, 10702-10707. http://dx.doi.org/10.1073/pnas.1014528108.

Akiyama, T., Osada, M., Isowa, M., Go, C.Y., Ochi, A., Elliott, I.M., Akiyama, M., Snead, O.C. Rutka, J.T., Drake, J.M., Otsubo, H., 2012. High kurtosis of intracranial electroencephalogram as a marker of ictogenicity in pediatric epilepsy surgery. Clin. Neurophysiol. Off. J. Int. Fed. Clin. Neurophysiol. 123, 93-99. http://dx.doi.org/10.1016/j.clinph. 2011.05.026.

Amunts, K., Kedo, O., Kindler, M., Pieperhoff, P., Mohlberg, H., Shah, N.J., Habel, U., Schneider, F., Zilles, K., 2005. Cytoarchitectonic mapping of the human amygdala, hippocampal region and entorhinal cortex: intersubject variability and probability maps. Anat. Embryol. (Berl.) 210, 343-352. http://dx.doi.org/10.1007/s00429-005-0025-5.

Arnulfo, G., Hirvonen, J., Nobili, L. Palva, S., Palva, J.M., 2015. Phase and amplitude correlations in resting-state activity in human stereotactical EEG recordings. NeuroImage 112, 114-127. http://dx.doi.org/10.1016/j.neuroimage.2015.02.031.

Ashburner, J., Friston, K.J., 2005. Unified segmentation. Neurolmage 26, 839-851. http:// dx.doi.org/10.1016/j.neuroimage.2005.02.018.

Atienza, M., Crespo-Garcia, M., Cantero, J.L., 2011. Semantic congruence enhances memory of episodic associations: role of theta oscillations. J. Cogn. Neurosci. 23, 75-90. http://dx.doi.org/10.1162/jocn.2009.21358.

Attal, Y., Schwartz, D., 2013. Assessment of subcortical source localization using deep brain activity imaging model with minimum norm operators: a MEG study. PLoS One 8, e59856. http://dx.doi.org/10.1371/journal.pone.0059856.

Attal, Y., Maess, B., Friederici, A., David, O., 2012. Head models and dynamic causal modeling of subcortical activity using magnetoencephalographic/electroencephalographic data. Rev. Neurosci. 23, 85-95. http://dx.doi.org/10.1515/rns.2011.056.

Bastin, J., Vidal, J.R., Bouvier, S., Perrone-Bertolotti, M., Bénis, D., Kahane, P., David, O. Lachaux, J.-P., Epstein, R.A., 2013. Temporal components in the parahippocampal place area revealed by human intracerebral recordings. J. Neurosci. 33, 10123-10131. http://dx.doi.org/10.1523/JNEUROSCI.4646-12.2013.

Bell, A.J., Sejnowski, T.J., 1995. An information-maximization approach to blind separation and blind deconvolution. Neural Comput 7, 1129-1159.

Bermudez-Rattoni, F., 2014. The forgotten insular cortex: its role on recognition memory formation. Neurobiol. Learn. Mem. 109, 207-216. http://dx.doi.org/10.1016/j.nlm. 2014.01.001.

Burke, J.F., Zaghloul, K.A., Jacobs, J., Williams, R.B., Sperling, M.R., Sharan, A.D., Kahana, M.J., 2013. Synchronous and asynchronous theta and gamma activity during episodic memory formation. J. Neurosci. 33, 292-304. http://dx.doi.org/10.1523/JNEUROSCI. 2057-12.2013.

Buzsáki, G., Moser, E.I., 2013. Memory, navigation and theta rhythm in the hippocampalentorhinal system. Nat. Neurosci. 16, 130-138. http://dx.doi.org/10.1038/nn.3304.

Cheyne, D., Bostan, A.C., Gaetz, W., Pang, E.W., 2007. Event-related beamforming: a robust method for presurgical functional mapping using MEG. Clin. Neurophysiol. Off. J. Int. Fed. Clin. Neurophysiol. 118, 1691-1704. http://dx.doi.org/10.1016/j.clinph.2007.05.064.

Clemens, Z., Borbély, C., Weiss, B., Erőss, L., Szűcs, A., Kelemen, A., Fabó, D., Rásonyi, G. Janszky, J., Halász, P., 2013. Increased mesiotemporal delta activity characterizes virtual navigation in humans. Neurosci. Res. 76, 67-75. http://dx.doi.org/10.1016/j. neures.2013.03.004

Cornwell, B.R., Johnson, L.L., Holroyd, T., Carver, F.W., Grillon, C., 2008. Human hippocampal and parahippocampal theta during goal-directed spatial navigation predicts performance on a virtual Morris water maze. J. Neurosci. 28, 5983-5990. http://dxdoi. org/10.1523/JNEUROSCI.5001-07.2008.

Crespo-Garcia, M., Cantero, J.L., Pomyalov, A., Boccaletti, S., Atienza, M., 2010. Functional neural networks underlying semantic encoding of associative memories. Neurolmage 50, 1258-1270. http://dx.doi.org/10.1016/j.neuroimage.2010.01.018.

Crespo-Garcia, M., Cantero, J.L., Atienza, M., 2012. Effects of semantic relatedness on agerelated associative memory deficits: the role of theta oscillations. NeuroImage 61, 1235-1248. http://dx.doi.org/10.1016/j.neuroimage.2012.03.034.

Dalal, S.S., Zumer, J.M., Guggisberg, A.G., Trumpis, M., Wong, D.D.E., Sekihara, K. Nagarajan, S.S., 2011. MEG/EEG source reconstruction, statistical evaluation, and visualization with NUTMEG. Comput. Intell. Neurosci. 2011, 1-17. http://dx.doi.org/10. $1155 / 2011 / 758973$

Dalal, S., Jerbi, K., Bertrand, O., Adam, C., Ducorps, A., Schwartz, D., Martinerie, J., Lachaux, J.-P., 2013. Simultaneous MEG-intracranial EEG: new insights into the ability of MEG 
to capture oscillatory modulations in the neocortex and the hippocampus. Epilepsy Behav. http://dx.doi.org/10.1016/j.yebeh.2013.03.012.

Dalal, S.S., Rampp, S., Willomitzer, F., Ettl, S., 2014. Consequences of EEG electrode position error on ultimate beamformer source reconstruction performance. Front. Neurosci. 8, 42. http://dx.doi.org/10.3389/fnins.2014.00042.

Duarte, I.C., Ferreira, C., Marques, J., Castelo-Branco, M., 2014. Anterior/posterior competitive deactivation/activation dichotomy in the human hippocampus as revealed by a 3D navigation task. PLoS One 9, e86213. http://dx.doi.org/10.1371/journal.pone.0086213.

Ekstrom, A.D., Caplan, J.B., Ho, E., Shattuck, K., Fried, I., Kahana, M.J., 2005. Human hippocampal theta activity during virtual navigation. Hippocampus 15, 881-889. http://dx. doi.org/10.1002/hipo.20109.

Fell, J., Ludowig, E., Staresina, B.P., Wagner, T., Kranz, T., Elger, C.E., Axmacher, N., 2011. Medial temporal theta/alpha power enhancement precedes successful memory encoding: evidence based on intracranial EEG. J. Neurosci. 31, 5392-5397. http://dx. doi.org/10.1523/JNEUROSCI.3668-10.2011.

Friese, U., Köster, M., Hassler, U., Martens, U., Trujillo-Barreto, N., Gruber, T., 2013. Successful memory encoding is associated with increased cross-frequency coupling between frontal theta and posterior gamma oscillations in human scalp-recorded EEG. NeuroImage 66, 642-647. http://dx.doi.org/10.1016/j.neuroimage.2012.11.002.

Gramfort, A., Papadopoulo, T., Olivi, E., Clerc, M., 2010. OpenMEEG: opensource software for quasistatic bioelectromagnetics. Biomed. Eng. Online 9, 45. http://dx.doi.org/10. 1186/1475-925X-9-45.

Greenberg, J.A., Burke, J.F., Haque, R., Kahana, M.J., Zaghloul, K.A., 2015. Decreases in theta and increases in high frequency activity underlie associative memory encoding. NeuroImage 114, 257-263. http://dx.doi.org/10.1016/j.neuroimage.2015.03.077.

Guderian, S., Schott, B.H., Richardson-Klavehn, A., Düzel, E., 2009. Medial temporal theta state before an event predicts episodic encoding success in humans. Proc. Natl. Acad. Sci. U. S. A. 106, 5365-5370. http://dx.doi.org/10.1073/pnas.0900289106.

Hafting, T., Fyhn, M., Molden, S., Moser, M.-B., Moser, E.I., 2005. Microstructure of a spatial map in the entorhinal cortex. Nature 436, 801-806. http://dx.doi.org/10.1038/ nature 03721

Hanslmayr, S., Spitzer, B., Bäuml, K.-H., 2009. Brain oscillations dissociate between semantic and nonsemantic encoding of episodic memories. Cereb. Cortex N. Y. N 1991 (19), 1631-1640. http://dx doi.org/10.1093/cercor/bhn197.

Hanslmayr, S., Staudigl, T., Fellner, M.-C., 2012. Oscillatory power decreases and long-term memory: the information via desynchronization hypothesis. Front. Hum. Neurosci. 6, 74. http://dx.doi.org/10.3389/fnhum.2012.00074.

Harris, K.D., Thiele, A., 2011. Cortical state and attention. Nat. Rev. Neurosci. 12, 509-523. http://dx.doi.org/10.1038/nrn3084.

Hasselmo, M.E., 2005. What is the function of hippocampal theta rhythm?-linking behavioral data to phasic properties of field potential and unit recording data. Hippocampus 15, 936-949. http://dx.doi.org/10.1002/hipo.20116.

Hillebrand, A., Barnes, G.R., 2002. A quantitative assessment of the sensitivity of wholehead MEG to activity in the adult human cortex. Neurolmage 16, 638-650.

Hölscher, C., Anwyl, R., Rowan, M.J., 1997. Stimulation on the positive phase of hippocampal theta rhythm induces long-term potentiation that can Be depotentiated by stimulation on the negative phase in area CA1 in vivo. J. Neurosci. 17, 6470-6477.

Huang, Y., Dmochowski, J.P., Su, Y., Datta, A., Rorden, C., Parra, L.C., 2013. Automated MRI segmentation for individualized modeling of current flow in the human head. J. Neural Eng. 10, 66004. http://dx.doi.org/10.1088/1741-2560/10/6/066004.

Jacobs, J., 2014. Hippocampal theta oscillations are slower in humans than in rodents: implications for models of spatial navigation and memory. Philos. Trans. R. Soc. Lond. Ser. B Biol. Sci. 369, 20130304 http://dx doi.org/10.1098/rstb.2013.0304.

Kaplan, R., Doeller, C.F., Barnes, G.R., Litvak, V., Düzel, E., Bandettini, P.A., Burgess, N., 2012. Movement-related theta rhythm in humans: coordinating self-directed hippocampal learning. PLoS Biol. 10, e1001267. http://dx.doi.org/10.1371/journal.pbio.1001267.

Kemp, A., Tischmeyer, W., Manahan-Vaughan, D., 2013. Learning-facilitated long-term depression requires activation of the immediate early gene, c-fos, and is transcription dependent. Behav. Brain Res. 254, 83-91. http://dx.doi.org/10.1016/j.bbr.2013.04. 036

Killian, N.J., Jutras, M.J., Buffalo, E.A., 2012. A map of visual space in the primate entorhinal cortex. Nature 491, 761-764. http://dx.doi.org/10.1038/nature11587.

Klimesch, W., Doppelmayr, M., Russegger, H., Pachinger, T., 1996. Theta band power in the human scalp EEG and the encoding of new information. Neuroreport 7, 1235-1240.

Larson, J., Munkácsy, E., 2015. Theta-burst LTP. Brain Res. 1621, 38-50. http://dx.doi.org/ 10.1016/j.brainres.2014.10.034.

Larson, J., Wong, D., Lynch, G., 1986. Patterned stimulation at the theta frequency is optimal for the induction of hippocampal long-term potentiation. Brain Res. 368, 347-350.

Lega, B.C., Jacobs, J., Kahana, M., 2012. Human hippocampal theta oscillations and the formation of episodic memories. Hippocampus 22, 748-761. http://dx.doi.org/10.1002/ hipo.20937.

Lega, B., Burke, J., Jacobs, J., Kahana, M.J., 2016. Slow-theta-to-gamma phase-amplitude coupling in human hippocampus supports the formation of new episodic memories. Cereb. Cortex N. Y. N 1991 (26), 268-278. http://dx.doi.org/10.1093/cercor/bhu232.

Libby, L.A., Hannula, D.E., Ranganath, C., 2014. Medial temporal lobe coding of item and spatial information during relational binding in working memory. J. Neurosci. 34, 14233-14242. http://dx.doi.org/10.1523/JNEUROSCI.0655-14.2014.

Litvak, V., Eusebio, A., Jha, A., Oostenveld, R., Barnes, G.R., Penny, W.D., Zrinzo, L., Hariz, M.I., Limousin, P., Friston, KJ., Brown, P., 2010. Optimized beamforming for simultaneous MEG and intracranial local field potential recordings in deep brain stimulation patients. Neurolmage 50, 1578-1588. http://dx.doi.org/10.1016/j.neuroimage.2009. 12.115 .

Long, N.M., Burke, J.F., Kahana, M.J., 2014. Subsequent memory effect in intracranial and scalp EEG. NeuroImage 84, 488-494. http://dx.doi.org/10.1016/j.neuroimage.2013. 08.052
Lorente de No, R., 1947. Action potential of the motoneurons of the hypoglossus nucleus. J. Cell. Physiol. 29, 207-287.

Maguire, E.A., 1997. Hippocampal involvement in human topographical memory: evidence from functional imaging. Philos. Trans. R. Soc. Lond. Ser. B Biol. Sci. 352 1475-1480. http://dx.doi.org/10.1098/rstb.1997.0134.

Maldjian, J.A., Laurienti, P.J., Kraft, R.A., Burdette, J.H., 2003. An automated method for neuroanatomic and cytoarchitectonic atlas-based interrogation of fMRI data sets. Neurolmage 19, 1233-1239.

Marchette, S.A., Vass, L.K., Ryan, J., Epstein, R.A., 2015. Outside looking in: landmark generalization in the human navigational system. J. Neurosci. 35, 14896-14908. http:/ dx.doi.org/10.1523/JNEUROSCI.2270-15.2015.

Merkow, M.B., Burke, J.F., Stein, J.M., Kahana, M.J., 2014. Prestimulus theta in the human hippocampus predicts subsequent recognition but not recall. Hippocampus 24 1562-1569. http://dx.doi.org/10.1002/hipo.22335.

Nadel, L., Hoscheidt, S., Ryan, L.R., 2013. Spatial cognition and the hippocampus: the anterior-posterior axis. J. Cogn. Neurosci. 25, 22-28. http://dx.doi.org/10.1162/jocn a_00313.

O'Keefe, J., Nadel, L., 1978. The Hippocampus as a Cognitive Map. Oxford University Press, Oxford, UK

Oostenveld, R., Fries, P., Maris, E., Schoffelen, J.-M., 2011. FieldTrip: open source software for advanced analysis of MEG, EEG, and invasive electrophysiological data. Comput. Intell. Neurosci. 2011. http://dx.doi.org/10.1155/2011/156869.

Osipova, D., Takashima, A., Oostenveld, R., Fernández, G., Maris, E., Jensen, O., 2006. Theta and gamma oscillations predict encoding and retrieval of declarative memory. J. Neurosci. 26, 7523-7531. http://dx.doi.org/10.1523/JNEUROSCI.1948-06.2006.

Pavlides, C., Greenstein, Y.J., Grudman, M., Winson, J., 1988. Long-term potentiation in the dentate gyrus is induced preferentially on the positive phase of theta-rhythm. Brain Res. 439, 383-387.

Pfurtscheller, G, 1992. Event-related synchronization (ERS): an electrophysiological correlate of cortical areas at rest. Electroencephalogr. Clin. Neurophysiol. 83, 62-69.

Pfurtscheller, G., Aranibar, A., 1977. Event-related cortical desynchronization detected by power measurements of scalp EEG. Electroencephalogr. Clin. Neurophysiol. 42, $817-826$.

Quraan, M.A., Moses, S.N., Hung Y., Mills, T., Taylor, M.J. 2011. Detection and localization of hippocampal activity using beamformers with MEG: a detailed investigation using simulations and empirical data. Hum. Brain Mapp. 32, 812-827. http://dx.doi.org/10. 1002/hbm.21068.

Santiuste, M., Nowak, R., Russi, A., Tarancon, T., Oliver, B., Ayats, E., Scheler, G., Graetz, G 2008. Simultaneous magnetoencephalography and intracranial EEG registration: technical and clinical aspects. J. Clin. Neurophysiol. Off. Publ. Am. Electroencephalogr. Soc. 25, 331-339. http://dx.doi.org/10.1097/WNP.0b013e31818e7913.

Schacter, D.L., Wagner, A.D., 1999. Medial temporal lobe activations in fMRI and PET studies of episodic encoding and retrieval. Hippocampus 9, 7-24. http://dx.doi.org/10. 1002/(SICI)1098-1063(1999)9:1<7::AID-HIPO2>3.0.CO;2-K.

Sederberg, P.B., Kahana, M.J., Howard, M.W., Donner, E.J., Madsen, J.R., 2003. Theta and gamma oscillations during encoding predict subsequent recall. J. Neurosci. 23 10809-10814.

Sederberg, P.B., Schulze-Bonhage, A., Madsen, J.R., Bromfield, E.B., McCarthy, D.C., Brandt A., Tully, M.S., Kahana, M.J., 2007. Hippocampal and neocortical gamma oscillations predict memory formation in humans. Cereb. Cortex N. Y. N 1991 (17), 1190-1196. http://dx.doi.org/10.1093/cercor/bhl030.

Stam, C.J., van Straaten, E.C.W., 2012. Go with the flow: use of a directed phase lag index (dPUI) to characterize patterns of phase relations in a large-scale model of brain dynamics. Neurolmage 62, 1415-1428. http://dx.doi.org/10.1016/j.neuroimage.2012.05.050.

Stam, C.J., Nolte, G., Daffertshofer, A., 2007. Phase lag index: assessment of functional connectivity from multi-channel EEG and MEG with diminished bias from common sources. Hum. Brain Mapp. 28, 1178-1193. http://dx.doi.org/10.1002/hbm.20346.

Staresina, B.P., Davachi, L., 2009. Mind the gap: binding experiences across space and time in the human hippocampus. Neuron 63, 267-276. http://dx.doi.org/10.1016/j.neuron. 2009.06.024.

Staudigl, T., Hanslmayr, S., 2013. Theta oscillations at encoding mediate the contextdependent nature of human episodic memory. Curr. Biol. CB 23, 1101-1106. http:/ dx.doi.org/10.1016/j.cub.2013.04.074

Summerfield, C., Mangels, J.A., 2005. Coherent theta-band EEG activity predicts itemcontext binding during encoding. NeuroImage 24, 692-703. http://dx.doi.org/10 1016/j.neuroimage.2004.09.012.

van Straaten, E.C.W., den Haan, J., de Waal, H., van der Flier, W.M., Barkhof, F., Prins, N.D. Stam, C.J., 2015. Disturbed phase relations in white matter hyperintensity based vascular dementia: an EEG directed connectivity study. Clin. Neurophysiol. Off. J. Int. Fed. Clin. Neurophysiol. 126, 497-504. http://dx.doi.org/10.1016/j.clinph.2014.05.018.

Van Veen, B.D., van Drongelen, W., Yuchtman, M., Suzuki, A., 1997. Localization of brain electrical activity via linearly constrained minimum variance spatial filtering. IEEE Trans. Biomed. Eng. 44, 867-880. http://dx.doi.org/10.1109/10.623056.

Vanderwolf, C.H., 1969. Hippocampal electrical activity and voluntary movement in the rat. Electroencephalogr. Clin. Neurophysiol. 26, 407-418.

Watrous, A.J., Fried, I., Ekstrom, A.D., 2011. Behavioral correlates of human hippocampal delta and theta oscillations during navigation. J. Neurophysiol. 105, 1747-1755. http://dx.doi.org/10.1152/jn.00921.2010.

Watrous, A.J., Lee, D.J., Izadi, A., Gurkoff, G.G., Shahlaie, K., Ekstrom, A.D., 2013. A comparative study of human and rat hippocampal low-frequency oscillations during spatial navigation: comparison of human and rodent theta. Hippocampus 23, 656-661. http://dx.doi.org/10.1002/hipo.22124.

Winson, J., 1978. Loss of hippocampal theta rhythm results in spatial memory deficit in the rat. Science 201, 160-163.

Zhang, H., Jacobs, J., 2015. Traveling theta waves in the human hippocampus. J. Neurosci. 35, 12477-12487. http://dx.doi.org/10.1523/JNEUROSCI.5102-14.2015. 